\title{
Behavioural GP Diversity for Adaptive Stock Selection
}

\author{
Wei Yan \\ Department of Computer Science \\ University College London \\ Gower Street, London, WC1E 6BT \\ w.yan@cs.ucl.ac.uk
}

\author{
Christopher D. Clack \\ Department of Computer Science \\ University College London \\ Gower Street, London, WC1E 6BT \\ c.clack@cs.ucl.ac.uk
}

\begin{abstract}
We present a new mechanism for preserving phenotypic behavioural diversity in Genetic Programming. We provide a real-world case study for hedge fund portfolio optimization, and experimental results on real-world data that indicate the importance of phenotypic behavioural diversity both in achieving higher fitness and in improving the robustness of the GP population for continuous learning.
\end{abstract}

\section{Categories and Subject Descriptors}

\section{I.2.M [Artificial Intelligence]: Miscellaneous}

\section{General Terms}

Algorithms, Experimentation

\section{Keywords}

Genetic Programming, Diversity, Phenotype, Finance, Robustness, Dynamic Environment

\section{INTRODUCTION}

In the context of our research into the applicability of Genetic Programming (GP) technology to the optimization of Hedge Fund portfolios, we have constructed an automated investment simulator that uses GP to derive a useful nonlinear relationship between a large number of factors relating to equities. This non-linear factor model assists the system in making buy/sell choices. Our system has been trained on a basket of 24 equities from the Malaysian stock market, and displays successful investment behaviour in out-ofsample tests. Having trained the system, our intention is that it should be used in a continuous learning mode [4] so that it can adapt to changes in the Malaysian economy by modifying (via retraining) the non-linear equation. However, standard GP systems are characterized by the fact that the final trained population often has little diversity and this could make it difficult to adapt to sudden changes in the economy.

Permission to make digital or hard copies of all or part of this work for personal or classroom use is granted without fee provided that copies are not made or distributed for profit or commercial advantage and that copies bear this notice and the full citation on the first page. To copy otherwise, to republish, to post on servers or to redistribute to lists, requires prior specific permission and/or a fee.

GECCO'09, July 8-12, 2009, Montréal Québec, Canada.

Copyright 2009 ACM 978-1-60558-325-9/09/07 ...\$5.00.
We have developed a new mechanism for preserving phenotypic behavioural diversity in a GP population, inspired by the risk-management practices of fund managers. In this paper we present that new system, together with the results of experiments to determine:

- the efficacy of the new system - to what extent it improves diversity, compared with standard GP;

- the impact of increased diversity on the ability of the GP population to adapt to a new environment; and

- the robustness of an individual chromosome obtained by such a system, compared with that obtained from a standard GP system - in particular, the fitness of a newly adapted individual after retraining on different amounts of data from a new economic environment.

\section{RELATED WORK}

Numerous techniques have been developed to maintain population diversity. These approaches have three main research directions:

1. the preservation of genotype diversity based on formallydefined structural distance measures;

2. the preservation of phenotype diversity based on the unique individual fitness in a population;

3. the reintroduction of genetic material in various evolutionary phases.

The majority of existing work on diversity aims to preserve genotype diversity using various measures of distance such as an edit distance [9] or a string edit distance for linear GP [1]. Monsieur and Flerackers [11] detect identical subtrees within a GP individual and delete individuals with low diversity and "DGEA" [16] uses a distance-to-averagepoint structure measure to alternate between decreasing and increasing diversity. Another popular technique is Fitness Sharing which smoothes the fitness landscape, initially used for maintaining diversity in genetic algorithms [6] though later applied to GP [5].

In contrast, there is very little work in the area of preserving phenotypic diversity. Two example techniques in this area are: (i) using entropy (the amount of disorder of the population) and free energy to measure phenotype diversity in terms of the number of unique fitness values in the population [13]; and (ii) using a selection method that is uniform over the fitness values [10]. 
Three examples of work where genetic material is introduced are: (i) random immigrants [7] where in every generation the population is partly replaced by randomly generated individuals; (ii) hypermutation [3], which increases mutation rate drastically whenever the average best individual performance worsens, and (iii) the Restart approaches [8], which advocate a complete restart of the GP to maintain diversity after a change in the environment has occurred.

\subsection{Limitations of related work}

None of the previously mentioned techniques are wholly satisfactory. Studies have shown that genotype diversity approaches "may not be useful for capturing the dynamics of a population" [2] and phenotypic measures appear to have better run performance. However, phenotype diversity techniques do not explicitly consider behavioural diversity of individuals, although they successfully spread individuals across different fitness levels. In other words, by ignoring the dynamic behaviour of individuals, the diversity in the same fitness level is not maintained. Legg and Hutter [10] remarked that "while the total population diversity was improved, the diversity among the fit individuals was not."

The reintroduction techniques also have their limitations: "random immigrants" and "hypermutation" do not perform well in continuously and abruptly changing environments, and "Restart" techniques ignore the knowledge accumulated by the population from previous learning. Restart techniques explicitly re-introduce diversity following a change in the environment, but may reconverge on a local optimum, especially when retraining only on data from the new environment where there are few new data points. Restart retraining on a mixture of old and new data suffers from the fact that it is very difficult to know a priori what mix of the two would give the best results [14].

Using any one technique on its own appears not to preserve useful diversity, implying that more elaborate techniques should be explored.

\subsection{Robustness}

The definition of robustness in evolutionary systems varies from author to author, but in broad terms it can be either robustness to internal changes (genotypic robustness) such as crossover and mutation [21], or robustness to external changes (phenotypic robustness). The latter can be either (i) robustness as the generalisation ability of GP-evolved programs [19]; (ii) robustness as the ability for self-repair when subject to severe phenotypic damage [18]; (iii) robustness as the ability to cope with non-constant noise [20]; or (iv) robustness as the sensitivity of performance quality in the presence of external environmental perturbations [17]. This last aspect is the most consistent with phenotypic robustness in nature. Although a biological system exhibits robustness in terms of genes, structures etc, only one measure of robustness matters: the ability to survive and reproduce when the environment changes adversely.

There are three ways we plan to measure robustness in the context of our finance application:

1. when exposed to volatile out-of-sample validation data, a more robust solution will have a lower standard deviation of returns (see Section 3), while those returns do not decrease;

2. when exposed to an out-of-sample volatile validation data-set, a more robust solution will have higher returns while the standard deviation does not increase.

3. when exposed to an out-of-sample volatile validation data-set, the mean return per unit of risk of a more robust solution will not significantly reduce from that measured during training.

\section{BEHAVIOUR DIVERSITY}

Consider two individuals that differ in the time-stepped fine detail of their dynamic behaviour over time-series training data. If that detailed behaviour were important for evolutionary selection, then it would be part of the calculation of fitness and individuals with different detailed behaviour would differ in fitness. However, where the time-stepped detail is irrelevant for selection, fitness can reflect general behaviour (over the whole time series) and two individuals who differ in detailed behaviour might have identical fitness. In this case, we propose that differences in detailed behaviour be used to preserve diversity of phenotypic behaviour. ${ }^{1}$

Given an individual that has a detailed phenotypic behaviour that is different from others of similar fitness: deletion of that individual would decrease the total population diversity of phenotypic behaviour. Conversely, if a set of individuals has entirely correlated behaviour and similar fitness then we can delete all but one from this set without comprising phenotypic behavioural population diversity.

Our new GP system preserves diversity at two levels:

1. simple phenotypic fitness diversity;

2. phenotypic behavioural diversity within groups of the same fitness.

For the former we hold individuals in fitness segments each segment contains individuals of similar fitness. For the latter, we establish the correlation of the phenotypic behaviour of all individuals in a segment, partition them into correlated groups and for each group we delete all but one individual - this is repeated for each segment.

In practice, determining the correlation coefficients of GP individuals is not always straightforward. However, in our financial portfolio application it is achieved by comparing the changes in Return on Investment (ROI) at particular time periods. If one individual's ROI drops and at the same time another individual's ROI increases at the same point in time in the training-data time series, then the two individuals are contra-correlated at that point. The degree of correlation can be calculated using a metric such as the Spearman Correlation test to compare behaviour at every time point - see Section 3.1. Note that the overall fitness for two individuals might be identical yet the ROI behaviours of the individuals might be contra-correlated.

\subsection{Description of the algorithm}

In our system, each individual has both a fitness value and an individual behaviour vector storing its historical behaviour (ROI performance). Let $O(I)$ be the vector of ROIs of an individual $I$ during the whole time period and $p_{\tau}(I)$ be the ROI of an individual at a particular time $\tau(1 \leq \tau \leq t)$. Then, $O(I)=\left\{p_{1}(I), \ldots, p_{t}(I)\right\}$. We call $O(I)$ the individual behaviour vector.

\footnotetext{
${ }^{1}$ Note that this is not a multi-objective GP system - diversity is not a separate objective.
} 
At the beginning of each evolutionary selection cycle, after the initial population fitness is calculated, we group individuals according to fitness value into $\kappa$ segments. Let $F_{\max }, F_{\min }$ be the maximum and minimum fitness values for a population. Segments $\left\{\left(F_{\text {min }}, F_{\text {min }}+\delta\right),\left(F_{\text {min }}+\delta+\right.\right.$ $\left.\left.1, F_{\text {min }}+2 \times \delta\right), \ldots,\left(F_{\min }+(\kappa-1) \times \delta+1, F_{\text {max }}\right)\right\}$ are collections of fitness intervals of equal length with defined lower and upper bounds (where $\left.\delta=\left(F_{\max }-F_{\min }\right) /(\kappa-1)\right)$.

Let average $S$ be a vector of average performance outputs per time period of all $n$ individuals $I_{S_{1}} \ldots I_{S_{n}}$ in a fitness segment $S$. We call average $S$ the "segment behaviour vector" given by average $S=\left\{\right.$ average $_{1}(S), \ldots$, average $\left.{ }_{t}(S)\right\}$ where average $_{t}(S)=\frac{1}{n} \sum_{i=1}^{n} p_{t}\left(I_{S_{i}}\right)$

Behavioural diversity in each of the fitness segments can be determined by measuring the correlation between an individual and its segment, as the segment behaviour vector should be representative of the general behaviour pattern of all the individuals in the segment. In this sense, if the difference of behaviour pattern between an individual and its segment is large, then it means that this individual exhibits unique or largely different behaviour from other individuals. We measure the behaviour relationship between the individual behaviour vector $O(I)$ and the segment behaviour vector average $S$ based on the Spearman correlation test. The Spearman correlation measure simply ranks the two variables, and makes no assumption about the distribution of the values. The Spearman correlation coefficient $\rho(O(I)$, average $S)$ is computed as follows: $1-\frac{6}{N^{3}-N} \sum_{i=1}^{N} d_{i}^{2}$ where $N$ is the numbers of pairs, and $d_{i}$ is the distance between (i) the rank of performance $p_{i}(I)$ (compared with all other $\left.p_{j}(I), j \neq i\right)$ in the individual behaviour vector and (ii) the rank of average $(S)$ (compared with all the other average $_{j}(S), j \neq i$ ) in the segment behaviour vector. The degree of correlation returned by this measure varies from -1.0 (perfect negative correlation), through 0.0 (no correlation), to 1.0 (perfect positive correlation). ${ }^{2}$

If a segment contains a set of individuals that have correlated or similar behaviour throughout the run, the behaviour diversity of the segment would be compromised without deleting individuals from this set. Conversely, if we keep an individual whose reactions to the environment are very different from other individuals, the diversity level of the segment can be maintained. Therefore, we first check for the correlation coefficient value $\rho$ of each individual in each segment and then we delete individuals with high $\rho$ (higher than a predefined correlation threshold, which we set empirically at 0.67 ). Additionally, in the case of domination of one or two particular fitness segment(s), which means that the number of individuals contained in the segment are higher than the average (defined as $P / s$ where $P$ is the total population size and $s$ the number of the segments), we delete not only those individuals with high correlation coefficient but also surplus individuals in the order of decreasing correlation. This algorithm encourages the creation of individuals at all fitness levels throughout the evolutionary cycle and also preserves diversity at the global population level. After

\footnotetext{
${ }^{2}$ Alternatively, measure the correlation between each pair of individuals in the same segment; in other words, create a correlation coefficient matrix for the segment. The major drawback of this approach is the computational cost $\left(O\left(n^{2}\right)\right.$ rather than $O(n))$.
}

Table 1: GP Parameter Settings

\begin{tabular}{|l|l|}
\hline Population size $(N)$ & 1000 \\
Method of generation & Ramped half and half \\
Function set & $\{+,-,, /$, Exp $\}$ \\
Terminal set & 20 firm-specific factors \\
Selection scheme & Random selection \\
Criterion of fitness & Annual Sharpe ratio \\
Trees generated by elitism & $10(1 \%)$ \\
Trees generated by crossover & $950(95 \%)$ \\
Trees generated by mutation & $40(4 \%))$ \\
Termination criterion & 100 -generation evolution \\
Max. depth initial generation & 6 \\
\hline
\end{tabular}

crossover and mutation, randomly generated individuals are inserted to keep the population size constant.

\section{HEDGE FUND SIMULATION}

To test the efficacy of the new diversity-preservation algorithm, we simulated a long/short market-neutral hedge fund of Malaysian equities. The GP system evolved a non-linear equation that used market data to determine whether each stock should be selected to buy, or to sell.

\subsection{System overview}

Our test system comprised a GP subsystem (see Table 1) utilizing the new diversity-preservation algorithm (we call this NGP), coupled with an investment simulator. The coupling between the two was the fitness function - the investment simulator was called each time the GP subsystem needed to determine the fitness of an individual, at which point the individual was used to control the simulation of an hedge fund of Malaysian stocks. The simulator was applied to training data giving monthly prices and other factors [22] for a period of 41 months. Monthly returns on investment were calculated, and at the end of each year the Sharpe ratio [15] was calculated. The simulator returned to the GP system both the fitness calculated from the Sharpe Ratio and a correlation vector of ROIs over the training period.

\section{Fitness}

The fitness $f$ for an individual is given by Equation 1 .

$$
f=\frac{1}{1-|1.5-S|} \quad \text { where } \quad S=\frac{1}{4} \sum_{i=1}^{4} \frac{\bar{x}_{i}-R F R_{i}}{\sigma_{i}}
$$

$S$ is the average Sharpe Ratio over the training period (comprising 4 sub-periods), $\bar{x}_{i}$ is the average monthly ROI over the sub-period $i, \sigma_{i}$ is the standard deviation of monthly ROIs over the sub-period $i$, and $R F R_{i}$ is the average monthly Risk Free Rate for sub-period $i$. We set $R F R_{i}$ to 0.003 for all $i$ (compounds to $3.6 \%$ per annum). Fund managers often set a target Sharpe Ratio, as do we - our target is 1.5, and the absolute difference between the measured Sharpe Ratio and the target is then normalized to provide a fitness that varies between 0 and 1 (the empirical range of $\mathrm{S}$ is $0.5<S<2.5$ ).

\section{Correlation}

The vector of ROIs returned by the simulator was used by the GP system to assess correlation with other individuals. This correlation data was then used to guide the preservation of phenotypic behavioural diversity (see Section 3.1). 


\subsection{The Investment Simulator}

We simulated a market-neutral long/short Hedge Fund of Malaysian equities. The fund focused on a basket of 24 Malaysian stocks, which it could buy ("go long") or sell (even if it didn't own any - "go short"). Since all the stocks in this basket were quite well correlated, the market-neutral strategy simply entailed buying the profitable stocks and selling short those stocks that were performing poorly.

The training data was monthly prices (and other technical and fundamental data) over a period of 41 months. Since we had only monthly data, all trading occured at the beginning of each month and the resulting stock mix was held for the duration of the month. At the beginning of each month, we used the individual provided by the GP system as a stock selection model that quantitatively measured the attractiveness of each stock; this model was a non-linear combination of technical and fundamental factors to predict the return expectation for each stock over a 4-week forward horizon.

For each month, we applied the stock selection model to the current month data - this was a table per stock with a total of 480 data points (20 factors $\times 24$ stocks). A return prediction was assigned to each stock.

Stocks were grouped into 4 sectors; within each sector all stocks were ranked according to expected return. The simulator then made the following fund management decisions:

- The long/short portfolio was dollar neutral and sector neutral. At all times, the portfolio held 24 stocks 12 long and 12 short, equally distributed across all sectors. The top 3 ranked stocks in each sector were bought (long) and the bottom 3 were sold (short).

- Sectors were equally weighted and each stock was given equal weight in the portfolio. Thus, each position accounted for approximately $4 \%$ of total portfolio value.

- We assumed $20 \%$ down-payment (margin) on each trade fully reconciled at the end of each month, $0.25 \%$ commission, and $5 \%$ financing rate for short trading.

At the end of each month, all of the positions held in the portfolio were closed and the profit or loss of the portfolio during the month was calculated. At the beginning of the next monthly trading cycle, the simulator updated the expected return based on the new "current" data and a new desired long/short portfolio was formed.

\section{METHOD}

The motivation for our research is to develop a system that can be used in a continuous-learning context [4], where the economic environment is dynamic and unpredictable (and so the most successful non-linear factor model will vary). Continuous learning in our context refers to retraining individuals whenever the market environment changes and a new batch of training data is available. The previously trained population is retained so that it can be retrained on this new data. Old data is not used for retraining.

Following a shift in economic context it will be necessary to continue to use the previously trained "best" individual while new data is being accumulated for retraining — we are interested in the behaviour of this previously-trained "best" solution, and how well it performs in the context of a new economic environment. When sufficient new data has been accumulated for re-training, we require that the system should re-train effectively on the new data - our expectation is that shifts in context will not normally be excessive and therefore retraining should start from the previously trained population (rather than from a random population) since the system is then less likely to converge on a local optimum, especially when there are few data points available for the new environment [12]. Initial training will use an "in-sample" data set: subsequent retraining will be on "out-of-sample" data in the context of the original training.

We performed experiments to answer the following three research questions:

1. does the new technique really improve population diversity when compared with a standard GP system, and how does this affect fitness?

2. when retraining on data that comes from a different economic context, how quickly and how well does the diverse population adapt to a new environment?

3. are trained individuals from the new system more robust when exposed to a new economic environment?

We ran three experiments, presented below. In each case, a standard GP system (SGP) was compared with our new GP system (NGP). The training data and validation data was in all cases identical for both SGP and NGP.

In-sample population dynamics (Experiment 1)

The aim of Experiment 1 was to see if NGP improved diversity, and what effect it had on fitness. Both SGP and NGP were trained separately using 41 months of data for 24 Malaysian stocks (from $31 / 7 / 97$ to $31 / 12 / 2000$ ). The following measurements were made each generation:

1. the fitness of the best chromosome, the fitness of the worst chromosome, the average fitness across all chromosomes, and the standard deviation of all fitnesses;

2. the distribution of chromosomes in the segment vector in terms of (i) fitness and (ii) phenotypic behaviour.

The average value from 5 runs of each measurement was plotted for each generation (see Fig. 1); the best and worst runs are indicated by error bars.

\section{Retraining population dynamics (Experiment 2)}

The aim of Experiment 2 was to see how well the population as a whole adapted to a new environment; in each case we started with a population that had previously been trained on the 41 months of training data (Experiment 1 above) and then retrained SGP and NGP separately on new training data that reflected a different economic climate. We used three sets of new training data and investigated the population behaviour for each:

Period a: $31 / 12 / 2000-31 / 4 / 2002$ (16 months).

Period b: $31 / 4 / 2002$ - 31/1/2003 (9 months)

Period c: $31 / 1 / 2003-31 / 12 / 2003$ (11 months)

As with Experiment 1, this experiment was repeated five times and for each generation the best, average and worst fitnesses, and standard deviation, were measured (see Figs. 4, 5, and 6). Convergence characteristics were compared by inspection of the graph. 


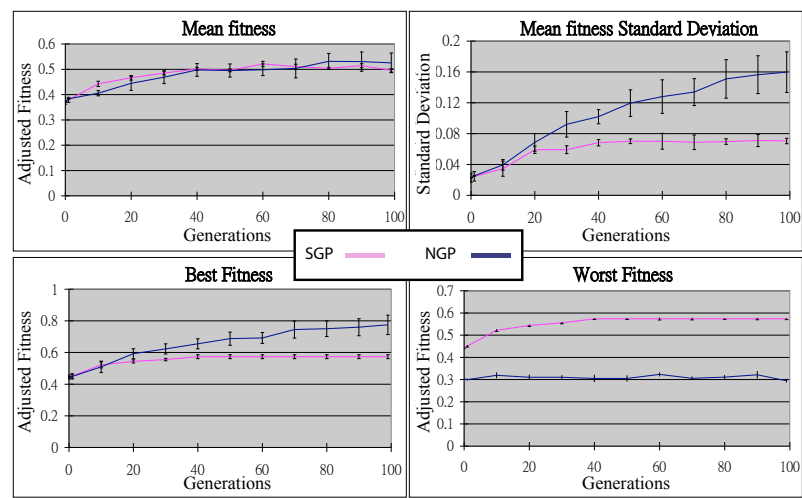

Figure 1: In-sample training (population dynamics).

Out-of-sample individual behaviour(Experiment 3)

The aim of Experiment 3 was to investigate whether improved population dynamics has resulted in improved robustness of behaviour of individuals in a previously unseen context using the three robustness measures in both a trainingvalidation context and a continuous-learning context. We used the following three sets of the data for this experiment. Period a: 31/07/1997 to 31/12/2000; Period b: 01/01/2001 to $31 / 06 / 2002$; Period c: $01 / 07 / 2002$ to $31 / 12 / 2003$.

1. Training-validation. Using the best individual trained using the Period a + Period $b$, a validation test was run on the Period c. First the best trained individual from the SGP system was used, and then the best trained individual from the NGP system.

2. Continuous-learning. For both SGP and NGP separately, starting with the previously trained population from Period a, we then retrained the population using Period b. The best individual was obtained and a validation test was run on Period c.

In all cases (for both SGP and NGP) the monthly ROI and monthly ROI standard deviation were measured and recorded, the Sharpe Ratio for the entire test period calculated, and the experiments repeated 25 times.

\section{DISCUSSION OF RESULTS}

\subsection{Behavioural Diversity Preservation}

\subsubsection{In-sample population dynamics}

Our first research question was "Does the new technique really improve population diversity when compared with a standard GP system, and how does this affect fitness?".

Fig.1 illustrates how the fitness of individuals in the population evolved over 100 generations while being trained on the base data of 41 months. Whilst the mean fitness difference between SGP and NGP is negligible, and remains negligible as the population evolves, the difference in standard deviation of fitnesses clearly increases as the population evolves, with NGP developing a substantially greater diversity of fitness. It is interesting to note a corollary of these two statements, which is that the fitness of the best individual is much greater for the NGP system - this in itself is a compelling reason to use diversity-preserving techniques, regardless of other possible benefits in terms of adaptability.

Fig. 2 gives the distribution of individuals across the fitness segments $(\kappa=5)$ at three points during evolution (after 0, 50 and 100 generations), indicating a slight bi-modal characteristic for both SGP and NGP. Of more importance from the point of view of phenotypic behavioural fitness, Fig. 3 investigates the correlation coefficient of individuals in the highest fitness segment (the segment from which the final solution(s) will be drawn). Fig. 3 illustrates how phenotypic behavioural diversity increases as the population evolves to help visualize this behaviour, on the left is a graph of correlations from a single (typical) run of NGP showing the mean, best and worst correlations of individual behaviour vectors with the segment behaviour vector; to quantify this behaviour, on the right is a graph comparing the mean correlations with error bars for 5 runs for NGP and SGP. ${ }^{3}$
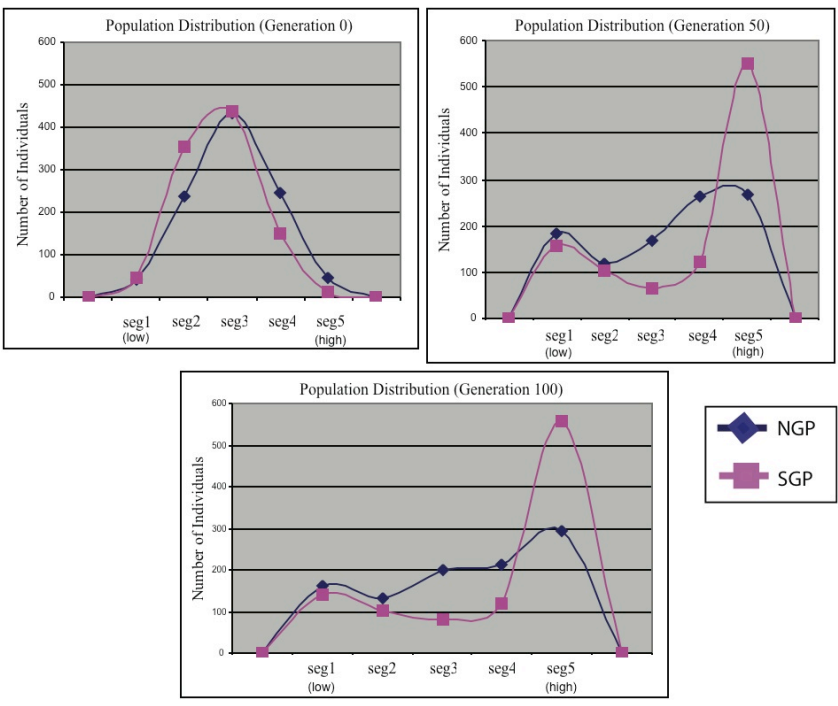

Figure 2: Phenotypic diversity for SGP and NGP

The results indicate that NGP provides a substantial improvement over SGP in diversity of phenotypic behaviour of the highest fitness segment in the later generations. It takes several generations for the diversity-preservation techniques to affect the highest-fitness segment - this explains why the graph on the right of Fig. 3 tracks SGP during the first 20 generations. Student's T-test (less than 0.00002 for Generations 80 and 100) confirms that for the later generations NGP provides a highly significant improvement.

\subsubsection{Retraining population dynamics}

Our second research question was "When retraining on data that comes from a different economic context, how quickly and how well does the diverse population adapt to a new environment?"

Figs. 4, 5 and 6 illustrate how the populations for SGP

${ }^{3}$ For SGP, we saved population snapshots for generations 0 , $20,50,80$ and 100, and identified which individuals would have been in the highest fitness segment if NGP were being run; then each such individual was used in the investment simulator and its individual behaviour vector obtained; finally, a segment behaviour vector was calculated and the correlations measured (repeated for each "snapshot"). 

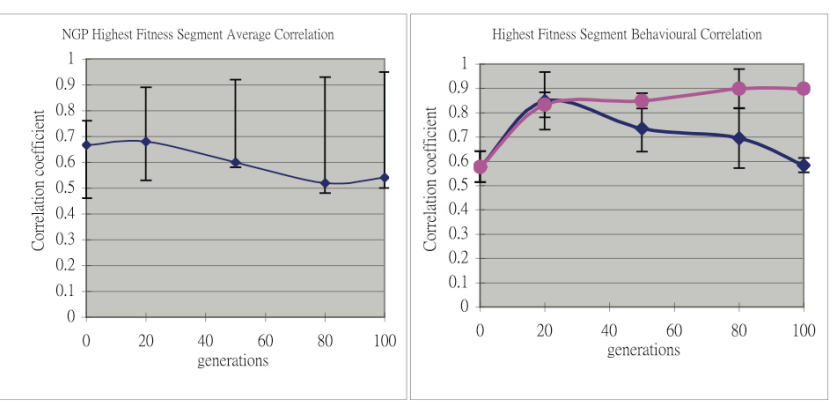

An example of a particular run of NGP

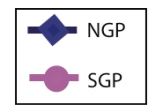

NGP vs SGP (multiple runs)

Figure 3: Evolution of behavioural phenotypic diversity for SGP and NGP

and NGP evolve during re-training on data from different economic climates. In all three cases (Periods a, b and c) the standard deviation of fitness for NGP is significantly better for NGP than for SGP after about 20 generations. NGP always starts with a "best" fitness that is higher than SGP (indicating that the NGP individual is already better able to cope with the new economic climate than the SGP individual), and that superiority is retained as the population retrains - in fact, in all cases NGP quickly finds the "ideal" Sharpe Ratio of 1.5 (giving the best adjusted fitness of 1.0) whereas SGP never finds that ideal individual.

When retraining, both SGP and NGP appear to converge on their respective "best" individuals at about the same rate, though SGP has the edge for Period c.

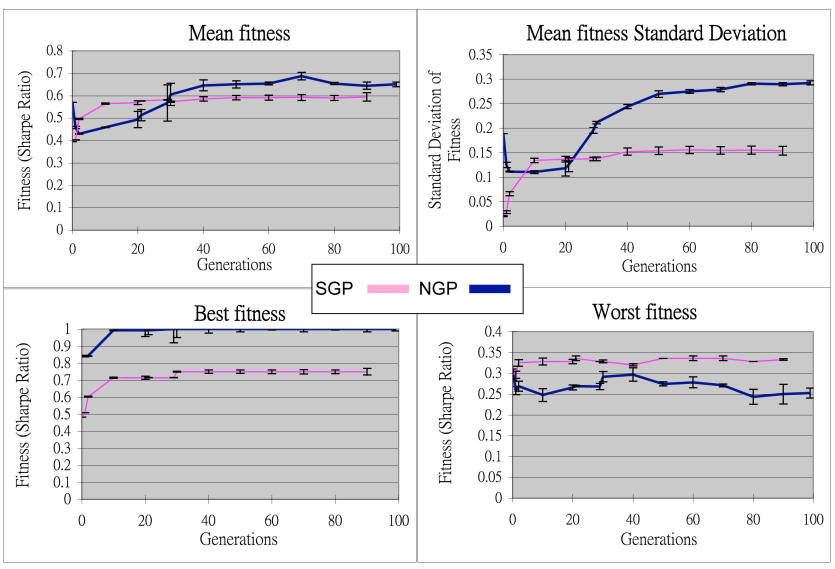

Figure 4: Retraining period-a population dynamics.

An interesting result from Figs. 4, 5 and 6 is the fact that the NGP standard deviation decreases for the first ten generations of re-training, then rises strongly. The initial generation comprises individuals trained for a different economic context, and these individuals do not have a normal distribution of fitnesses in the context of the new data. In particular, (i) the fitness peak for the highest segment that is observed at the end of the original training is unlikely to occur in the context of the new data, and it is much more likely that a peak will occur in the middle segments, and (ii) the behavioural diversity of individuals in the highest seg-

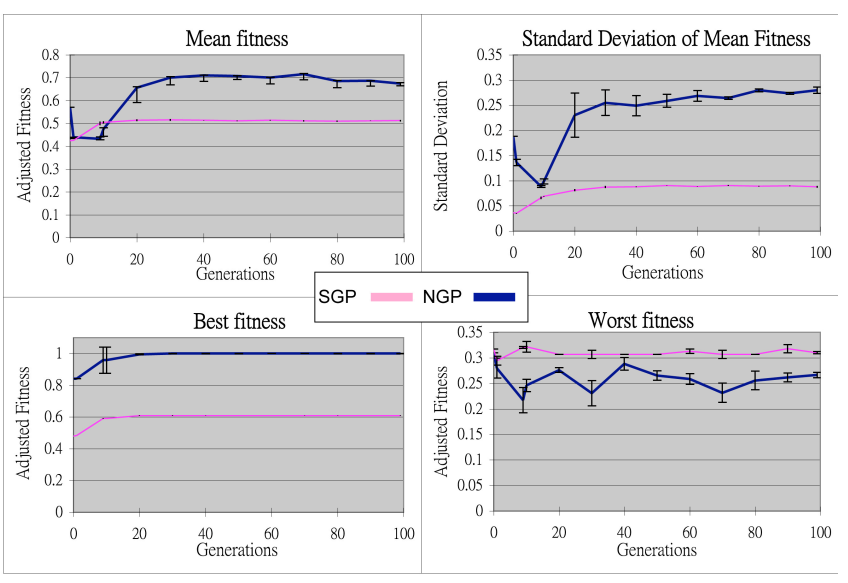

Figure 5: Retraining period-b population dynamics.

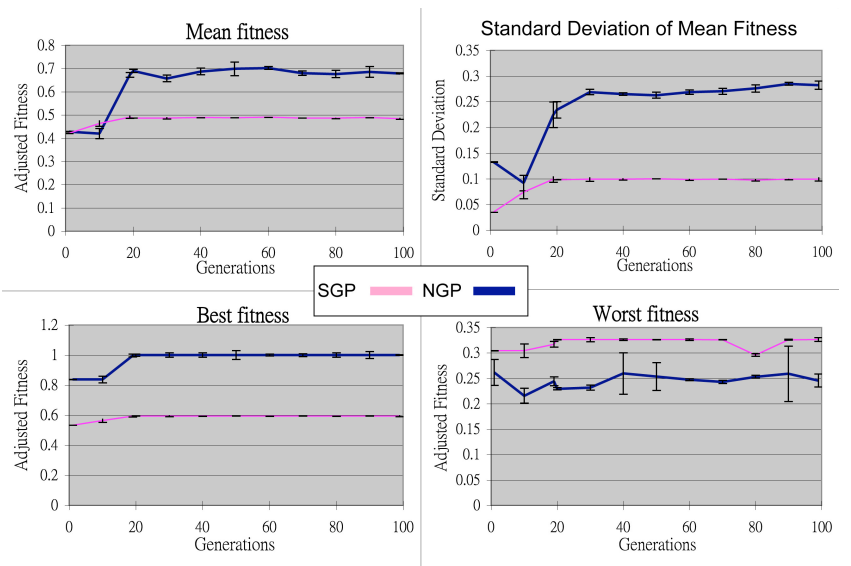

Figure 6: Retraining period-c population dynamics.

ment is likely to be low. In the early generations, the NGP algorithm will discard many individuals whose behaviours are too well correlated with others, and replacements will be drawn from random samples - the overall effect will be to increase the numbers of individuals in the middle segments (i.e. producing a more peaked distribution). The NGP standard deviation therefore dips towards that of SGP, then rises strongly after the distribution has normalised and the diversity-preserving algorithm has a stronger effect.

\subsubsection{Out-of-sample individual robustness}

Our third research question was "Are trained individuals resulting from the new system more robust when exposed to a new economic environment?"

Case 1. The best individual trained from 31/07/1997 to $31 / 06 / 2002(\operatorname{period} a+b)$ and tested over the pe$\operatorname{riod} 01 / 07 / 2002$ to $31 / 12 / 2003(\operatorname{period} \mathrm{c})$.

Fig. 7 and Fig. 8 illustrates the performance robustness of NGP and SGP using robustness measures 1, 2 and 3 when the economic context changes.

1. Standard deviation of NGP and SGP is almost on the same level (a ranked T-test indicates no significant difference in the distribution). This indicates that NGP and SGP achieves similar degree of robustness in terms of standard deviation of returns (measure 1). 
2. However, NGP consistently generates considerably higher average returns than SGP. This indicates that NGP achieves superior degree of robustness in terms of average returns (robustness measure 2).

3. SGP still outperforms the index portfolio. Although SGP and the index portfolio produces similar level of returns, SGP has lower return volatility.

4. The Sharpe Ratio over the test period is also calculated and compared. The percentage reductions in Sharpe Ratio from in-sample training to out-of-sample validation of NGP is less significant than SGP. The reduction for NGP is $32 \%$ (T-test $t(30)=8.41, p=$ $5.3 \times 10^{-11}$ ) and for SGP is $57 \%$ (T-test $t(30)=9.90$, $\left.p=3.4 \times 10^{-13}\right)$. NGP is more robust in terms of robustness measure 3 .

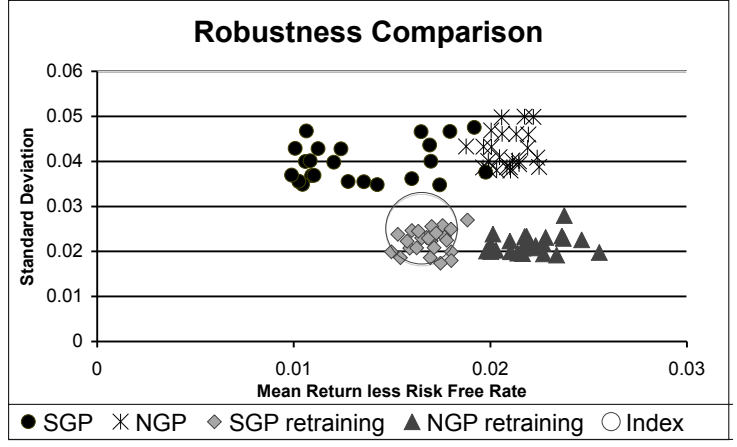

Figure 7: Robustness Comparison (Cases 1 \& 2)

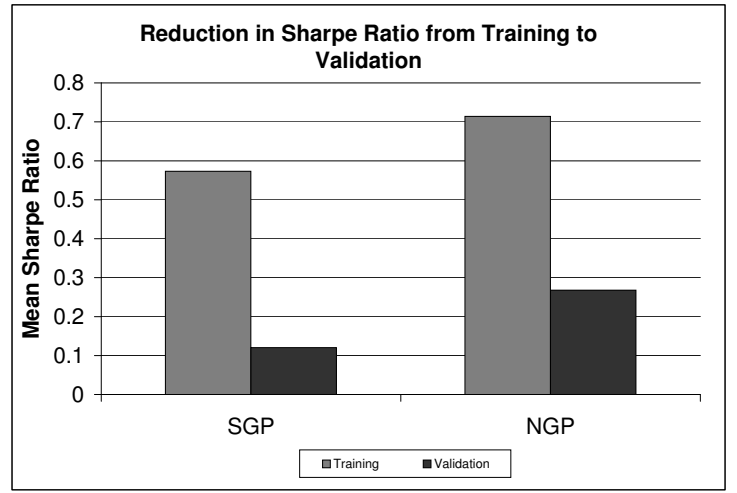

Figure 8: Robustness (Sharpe Ratio)

Case 2. Retraining was performed on the last generation of individuals initially trained on Period a. After retraining on Period $b$ (only), the best individual was tested on Period c. The monthly ROI and monthly

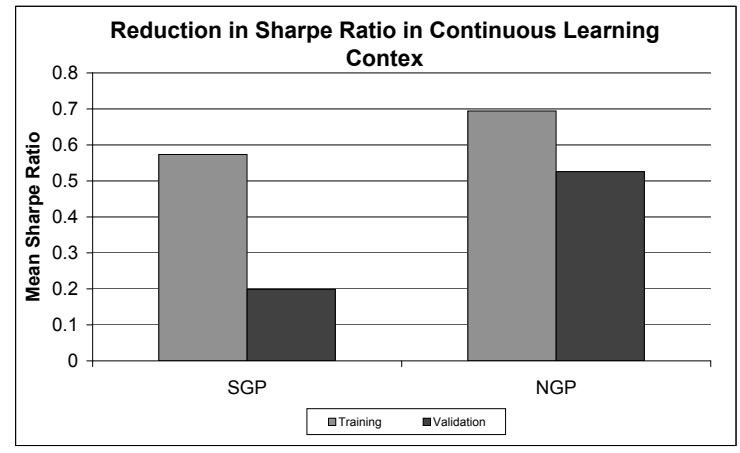

Figure 9: Retraining Robustness (Sharpe ratio)

standard deviation of ROI in the new economic climate is plotted for both the best NGP individual and the best SGP individual. Retraining was carried out on 18 months data and tested on remaining 18 months data. This simulated a continuous-learning context where the GP system is periodically retrained to accomodate non-extreme changes in the economy.

1. Fig. 7, the comparison of robustness plot indicates that NGP in the continuous-learning context can produce more robust individuals than SGP in terms of average return (measure 2). NGP and SGP share a similar level of volatility but the mean returns of NGP are clearly greater than those of SGP. This observation is consistent with Case 1. (robustness measure 2)

2. As in Case 1, the Sharpe ratios over the test period are calculated and compared. Fig. 9 shows that NGP $\left(-24 \%, t(30)=7.28, p=3.9 \times 10^{-9}\right)$ suffers much less the percentage reduction in Sharpe ratio from training to out-of-sample than SGP $(-65 \%, t(30)=8.89, p=$ $\left.6.3 \times 10^{-11}\right)$. NGP is more robust in terms of degree of change in Sharpe ratio. (robustness measure 3)

Traditional training and validation vs Continuous learning (Case 1 vs Case 2)

Fig. 7 compares robustness of the trained individuals obtained through the traditional training and validation method and the continuous learning method. T-test comparison (Table 2) of return and standard deviation indicates a statistically significant difference between individual robustness evolved by systems with retraining and systems without retraining. Individuals evolved by NGP with retraining achieve significantly lower standard deviation of ROI than those evolved by NGP with traditional training and validation method, while maintaining comparable level of return. Very similar observation can be applied when comparing individuals evolved by SGP with retraining against individuals evolved by SGP with traditional training and validation. Therefore, the continuous learning method generates more robust individuals by robustness measure 1 . 
Table 2: Summary of Ranked T-test (p-values)

\begin{tabular}{|l|c|c|}
\hline & Std. Devs. & ROIs \\
\hline SGP retraining vs. SGP: & $3.06 \times 10^{-16}$ & 0.28 \\
\hline NGP retraining vs. NGP: & $3.24 \times 10^{-16}$ & 0.42 \\
\hline
\end{tabular}

\section{CONCLUSION}

In a highly dynamic environment where the fitness landscape displays some exploitable degree of similarity before and after changes, it is possible to improve robustness of the solution by preserving the behavioural diversity of GP population. Our results provide an insight into the utility of the new algorithm (NGP) compared with a standard GP algorithm (SGP). On our test data:

1. using robustness measure 2, NGP consistently produced a "best" individual more robust with higher fitness than SGP without increasing volatility than SGP.

2. using robustness measure 3, NGP consistently produced a "best" individual more robust with lower rate of reduction of Sharpe ratio from training to out-ofsample test than SGP.

3. in continuous adapation to a changing environment, NGP consistently produced a "best" individual more robust than SGP using robustness measures 2 and 3.

4. NGP consistently retrained (in the context of a shift in the environment) faster and better than SGP.

Our results also confirm that, on our test data, the new algorithm increases population diversity of standard fitness and diversity of phenotypic behaviour. Analysis indicates that the difference from a standard GP algorithm is statistically highly significant. Moreover, our results indicate that a continuous learning method via retraining can generate more robust individuals in changing environments.

We conclude that (i) there is good evidence to recommend the use of NGP for preserving diversity of phenotypic behaviour in a GP context which uses time-series data, where an individual's phenotypic behaviour can be defined and measured, and where the environment is highly dynamic; and (ii) there is encouraging evidence to recommend the use of NGP for improving robustness in continuous adaptation contexts where the environment is continually changing. Further work is now required: to investigate the use of different correlation measures; to undertake a parameter sensitivity analysis; to establish repeatability for a much wider range of test cases and over more runs; and to obtain empirical data from a continuous-learning system.

\section{REFERENCES}

[1] M. Brameier and W. Banzhaf. Explicit control of diversity and effective variation distance in linear genetic programming. LNCS, 2278:37-49, 2002.

[2] E. K. Burke, S. M. Gustafson, G. Kendall, and N. Krasnogor. Advanced population diversity measures in genetic programming. $L N C S$, 2439:341-350, 2002.

[3] H. Cobb. An investigation into the use of hypermutation as an adaptive operator in genetic algorithms having continuous, time-dependent nonstationary environments. Technical report, Naval Research Laboratory, 1990.

[4] M.H. Dempster and C.M. Jones. A real-time adaptive trading system using genetic programming. Quantitative Finance, Vol 1, 2001.

[5] A. Ekart and S. Nemeth. Maintaining the diversity of genetic programs. In Proceedings of the 5th European Conference, Genetic Programming, LNCS, 2278:162-171. Springer-Verlag, 2002.

[6] D. Goldberg. Genetic Algorithms in Search, Optimisation, and Machine Learning. Addison-Wesley, 1989.

[7] J. Grefenstette. Genetic algorithms for changing environments. In Parallel Problem Solving from Nature 2, pages 137-144. North Holland, 1992.

[8] C. Karr. Genetic algorithms and fuzzy logic for adaptive process control. In Intelligent Hybrid Systems, Wiley, 1995.

[9] R. Keller and W. Banzhaf. Explicit maintenance of genetic diversity on genospaces. Unpublished Manuscript, 1994.

[10] S. Legg and M. Hutter. Fitness uniform deletion: A simple way to preserve diversity. In Proc. GECCO'05, pages 1271-1278. ACM, 2005.

[11] P. Monsieur and E. Flerackers. Increasing the diversity of a population in genetic programming. In Proc. GECCO'01, page 185. Morgan Kaufmann, 2001.

[12] C. Reeves and H. Karata. Dynamic sequencing of a multi-processor system: a genetic algorithm approach. In Artificial Neural Nets and Genetic Algorithms. Springer, 1993.

[13] J. Rosca. Entropy-driven adaptive representation. In Proc. Workshop Genetic Programming: From Theory to Real-World Applications, pages 23-32, 1995.

[14] C. Schoreels and J. M. Garibaldi. The effect of varying parameter on performance for adaptive agents in techical equity market trading. In Proc. ICCC, 2005.

[15] W. F. Sharpe. The sharpe ratio. J. Portfolio Management, 21:49-58, 1994.

[16] R. K. Ursem. Diversity-guided evolutionary algorithms. LNCS, 2439:462-474, 2002.

[17] J. Herrmann. A genetic algorithm for minmax optimisation problems. Proc. CEC'99, vol. 2, pages 1099-1103. IEEE, 1999.

[18] J. F. Miller. Evolving a self-repairing, self-regulating, french flag organism. LNCS, 3102:129-139, 2004.

[19] F. W. Moore and O. N. Gacia. A new methodology for reducing brittleness in genetic programming. In Proc. NAECON'97, 1997.

[20] V. Nissen and J. Propach. On the robustness of population-based versus point-based optimisation in the presence of noise. IEEE Transactions on Evolutionary Computation, 2(3), 1998.

[21] T. Soule. Operator choice and the evolution of robust solutions. In Genetic Programming Theory and Practice, Chapter 16, pages 257-270. Kluwer, 2003.

[22] W. Yan and C. D. Clack. Evolving robust GP solutions for hedge fund stock selection in emerging markets. In Proc. GECCO '07, 2:2234-2241. ACM, 2007. 